\title{
Rapid Deployment ECMO
}

\author{
Roberto Chiletti, MD, FCICM ${ }^{1}$ \\ Warwick Butt, MBBS, FRACP, FCICM 1,2 \\ Graeme MacLaren, MBBS, FCICM, FCCM ${ }^{1,2,3, *}$
}

\author{
Address \\ *,1Paediatric Intensive Care Unit, Royal Children's Hospital, \\ Flemington Rd, Parkville, Melbourne, VIC 3052, Australia \\ Email: gmaclaren@iinet.net.au \\ ${ }^{2}$ Department of Paediatrics, University of Melbourne, Melbourne, Australia \\ ${ }^{3}$ Cardiothoracic Intensive Care Unit, National University Health System, \\ Singapore, Singapore
}

Published online: 25 January 2015

(C) Springer International Publishing AG 2015

This article is part of the Topical Collection on Pediatric Critical Care Medicine

Keywords Extracorporeal membrane oxygenation - Extracorporeal life support - ECPR - Cardiac arrest

\section{Opinion statement}

In the last two decades, technological advances in equipment and improvements in both safety and patient survival have led to extracorporeal membrane oxygenation (ECMO) being increasingly used for a wide range of conditions causing cardiorespiratory failure. Earlier recognition of the deteriorating patient and use of ECMO to reverse multiorgan failure and prevent sudden cardiac arrest is associated with improved outcomes. As mortality and long-term disability remain high after cardiac arrest in children, ECMO CPR (ECPR) has been utilized to reverse the sudden loss of cardiac output after unanticipated cardiorespiratory events. To ensure not only survival but also the absence of major morbidity after ECPR, future research should focus on preventative resuscitation, further investigating the natural history of the underlying conditions and minimizing the complications of ECMO.

\section{Introduction}

In pediatric critical care, low cardiac output state (LCOS) and cardiogenic shock, sepsis, and acute respiratory distress syndrome (ARDS) are all conditions that can lead to multiorgan dysfunction and subsequent cardiorespiratory arrest, with substantial morbidity and mortality $[1,2]$.

Extracorporeal membrane oxygenation (ECMO) siphons venous blood out of the patient, oxygenates it, clears carbon dioxide, and then pumps it back in. It can provide complete cardiorespiratory support and reestablish end-organ perfusion and oxygen delivery. In the early days of ECMO, this support was considered experimental with very strict indications and length of support. The last 15 years have been rich in technological advances, experience sharing through single center reports, and thorough analyses of the registry of the Extracorporeal Life Support Organization (ELSO), together with improvement in patient and circuit management. Despite a lack of extensive grade I evidence, ECMO has become a standard of care in pediatrics to support respiratory and/or cardiac function when conventional medical treatment is failing. Venovenous 
ECMO (VV-ECMO) supports lung function by oxygenating venous blood, reduces or obviates the risk of ventilator-induced lung injury, corrects respiratory acidosis by removing carbon dioxide, and indirectly improves cardiac function through reducing intra-thoracic pressure, reducing pulmonary vascular resistance and oxygenating coronary arterial blood. Venoarterial ECMO (VA-ECMO) provides full cardiac and respiratory support ensuring adequate perfusion pressure and oxygen delivery to organs and allowing additional cardiac rest and recovery $[3,4]$. A cardiac arrest represents the most extreme form of cardiopulmonary failure and carries high morbidity and mortality $[5,6 \bullet]$.

Extracorporeal cardiopulmonary resuscitation (ECPR) is the use of ECMO during cardiac arrest. Several levels of intervention are required to establish ECMO support during cardiac arrest. This review will outline the indications for pediatric ECPR, the necessary components of an ECPR program, and the outcomes in selected patient populations.

\section{In-hospital versus out-of-hospital arrest}

In children, morbidity and mortality after cardiac arrest remain high, with better survival rates in in-hospital cardiac arrest (IHCA) versus out-of-hospital cardiac arrest (OHCA). In the past three decades, rates of survival to hospital discharge for pediatric IHCA have progressively improved from $9 \%$ in the 1980 s [7] to $35 \%$ in the 2000s [6•]. In the 2013 report from the American Heart Association, ECPR was used in $10.5 \%$ of the patients with a reported acute resuscitation survival rate of $34.3 \%(\mathrm{RR}, 1.22)$ but lower longer term survival [6•]. In the same year, a study of pediatric OHCA demonstrated survival rates of $5.4 \%$ [8]. In the large 2013 multicenter observational prospective study [5], greater mortality after IHCA was seen in patients with malignancy and those requiring inotropic support. Other risk factors for poor outcomes included the location of cardiac arrest (with emergency departments being worse than intensive care units), epinephrine doses, bicarbonate administration, and volume expansion. The nonROSC (return of spontaneous circulation) and death rate increased as duration of CPR increased; CPR more than 10 min was a risk factor for mortality [5]. Similar findings were shown in another study, where longer duration of CPR before ECMO was associated with worse survival and neurological outcome [9•]. However, this is not a consistent observation $[10,11]$ and suggests that the quality of CPR rather than its duration before
ECMO support may be more important. In a subgroup analysis of a larger study, survival after ECPR for more than 35 min was $33.3 \%$, compared to $12.5 \%$ if CPR was for more than $35 \mathrm{~min}$ without the use of ECMO, with lower survival in the general (surgical and medical) patient group compared to the cardiac patients; neurological outcome did not differ between the two groups [12].

Other factors associated with worse outcomes include higher lactate and lower $\mathrm{pH}$ levels before ECPR $[10,13,14]$. Renal dysfunction pre- or post-ECPR as well as the need for renal replacement therapy (RRT) during ECMO have been linked to poor outcome [15-18]. These additional factors stem from more prolonged periods of inadequate end-organ perfusion and poor oxygen delivery-perhaps in patients in refractory shock, which culminates in cardiac arrest, for example. In addition to prompt, high-quality CPR, earlier deployment of ECMO or more aggressive conventional care might lead to better outcomes.

The bleaker outcomes from OHCA may be explained by these factors, as the likelihood of prompt, effective CPR is reduced. Witnessed arrest, older age, and shockable rhythm at presentation have been associated with better long-term survival [8]; no survival was reported for CPR more than $31 \mathrm{~min}$ in emergency department (ED) and more than three doses of epinephrine [19]. Some adult centers have started offering ECPR for OHCA patients, but data on this practice in pediatrics are very limited [20•, 21-23]. A metanalysis in 2011 of OHCA and ECMO revealed that ECPR provided higher survival

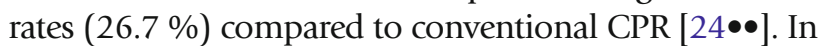
a large prospective multicenter study in Japan (SAVE-J study), neurological outcomes after OHCA at 1 and 6 month was better for the ECPR group rather than conventional CPR (12.3 vs. $1.5 \%$ and 11.2 vs. $2.6 \%$, respectively) [25]. It is difficult to generalize the Japanese experience to the rest of the world and even harder to extrapolate it to a pediatric population. A large number of patients had witnessed arrests $(90.7 \%)$ and received bystander CPR (71.8 \%). In the SAVE-J study, patients in ventricular tachycardia or fibrillation were selected, who have better survival rates when compared to patients presenting with nonshockable rhythms (70.8 vs. $36.8 \%$ ) $[24 \bullet \bullet, 25]$. However, the cause of pediatric OHCA is rarely primary cardiac, but rather respiratory with secondary hypoxia, with consequently higher risks of hypoxic brain injury during CPR and worse long-term outcomes. We do not recommend ECPR for pediatric OHCA except in cases of witnessed arrest with immediate, good quality $\mathrm{CPR}$ and evidence of adequate end-organ perfusion (e.g., 
low lactate on arrival and equal and reactive pupils) or with intermittent ROSC. ECPR could also be considered in nonasphyxial accidental hypothermia $\left(<28{ }^{\circ} \mathrm{C}\right)$; ECPR can facilitate rapid rewarming up to $33{ }^{\circ} \mathrm{C}$ with subsequent slow rewarming to normothermia. Adverse prognostic factors in this setting include $\mathrm{pH}<6.5$, hypercarbia, and hyperkalemia on arrival [26•, 27-29].

\section{Technology}

Rapid deployment of ECMO is now possible because of recent technological advances in circuit design. The ideal circuit has small priming volumes, is quick to prime or can be preprimed, and has minimal sites of stasis and turbulence. All of these factors reduce both the risk of clot formation and the inflammatory response due to contact of blood with a nonbiological surface [30-32]. They facilitate rapid priming and utilization of ECMO, with reduced complications and improved outcomes $[9 \bullet]$. One area of controversy in ECMO concerns what constitutes the best type of pump head $[33,34]$ : Roller pumps are large, dependent on gravity to function properly, and are not particularly portable. Tubing rupture is a rare but substantial complication. On the other hand, centrifugal pumps are much smaller but can lead to hemolysis and complications related to the negative pressure they generate $[31,35 \bullet, 36,37]$. Many high volume centers will keep preprimed circuits for up to 30 days; this allows a stand-by ECMO machine at any time without an apparent increase in the risk of infection [38, 39•] (Fig. 1).

\section{ECLS programs and rapid response teams}

Pediatric cardiac arrest is clearly a very stressful situation for clinicians and families, perhaps even more so when the decision is made to use ECMO. In 1998, a report of the Boston experience demonstrated that a rapid response team could achieve cannulation and initiate ECMO within $15 \mathrm{~min}$ from the call-out, with improved survival to $64 \%$ [40]. Several subsequent reports from different centers around the world have followed a similar model, in which a rapid response team (RaRT) is alerted in the setting of cardiac arrest or emergent cannulation $[40,41,42 \bullet, 43 \bullet \bullet, 44 \bullet]$. The members of the RaRT have predefined roles and generally train with medical simulation to improve performance [45]. Different institutions have different members as part of their RaRT (i.e., general surgeons, cardiac surgeons, ECMO nurses, respiratory technologists, intensivists, and perfusionists), but there is still variation in the immediate availability of team members, whether staff
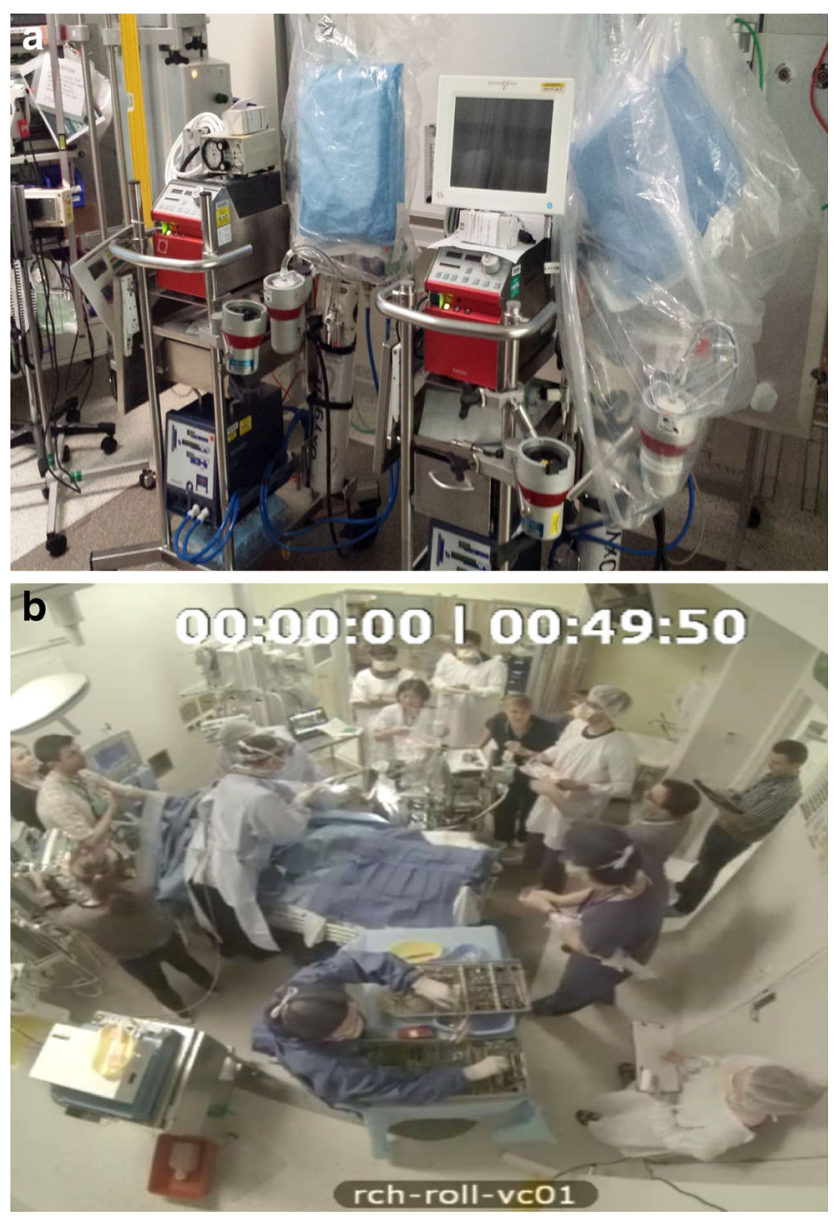

Fig. 1. a ECMO circuits pre-primed, sterile, and ready to use. These can be kept on stand-by for at least 30 days. b An ECPR simulation in progress. Larger ECLS programs often use simulation to maintain skills, team work, and in-house credentialing. (Courtesy of Amy Johansen, Royal Children's Hospital, Melbourne, Australia).

are in-house or on-call from home. A study of ECPR found a reduction in neurological complications after implementation of a RaRT but with no difference in mortality [42•]. In another small, single center study, the survival to hospital discharge after ECPR was high (57\%) in the absence of a RaRT [46]. Team composition varies but should include a person able to cannulate, a person dedicated to the ECMO circuit and pump and a person capable of helping as needed; the rest of the resuscitation team can then focus on the patient. It is important to establish an ECLS program with clear direction, protocols, continuous education, and quality 
assurance $[3,47 \bullet \bullet]$. Although higher center volume has been associated with better survival, it is unlikely that center volume is the sole determinant of the quality of a program $[48,49 \bullet]$.

\section{Cannulation}

The type of cannulation strategy is determined by many factors, but is almost always VA, and the goal is partial support with cannulae that are easy to insert, rather than the largest available size. A very important factor is whether or not the event is in patients after cardiac surgery, where usually a clear hospital policy is in place. The cardiac surgeon will use either a central or peripheral approach. For patients who have acquired cardiac disease or cardiac arrest due to sepsis or drug overdose peripheral VA cannulation will usually be performed.

In patients following cardiac surgery, sternotomy and central cannulation are the fastest means of initiating ECMO. This allows the largest cannulae to be inserted and facilitates the highest blood flows. Limitations of central cannulation for ECPR include the need to interrupt chest compressions and the subsequent risk of bleeding and infection. Peripheral cannulation generally remains the best modality for noncardiac surgical patients, with jugular vein and carotid artery cannulae for children $<15 \mathrm{~kg}$ and femoral artery and vein cannulae for children above $15 \mathrm{~kg}[50,51]$. However, in a recent review of the ELSO registry, carotid artery cannulation was associated with a higher risk of neurological complications and longer duration of mechanical support when

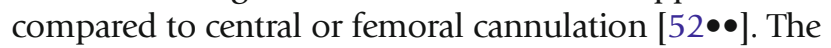
risks of femoral cannulation include more frequent pulmonary and gastrointestinal bleeding, limb ischemia, and differential cyanosis, where deoxygenated blood is ejected out of the systemic ventricle into the coronary and cerebral arteries, while blood from the ECMO circuit perfuses only the descending aorta. Specific cannulation strategies have been considered in the setting of:

- Pulmonary hypertension (neck cannulation in order to minimize cerebral desaturation) [51]

- Fulminant sepsis (central cannulation to allow very large venous and arterial cannula and hence very high blood flow rates of $150+$

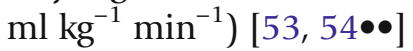

- Single ventricle anatomy with cavopulmonary anastomoses (bicaval or central cannulation, or takedown of the cavopulmonary anastomoses) $[51,55,56 \bullet]$.
Cannulation strategies may change throughout an ECMO run, based on recovery of cardiac or pulmonary function and evolution of the disease, such as conversion from VA- to VV-ECMO (in ECPR for fulminant bacterial pneumonia, cardiac recovery can occur within 3-5 days, but pulmonary recovery may be substantially slower over 2 weeks), short term centrifugal VAD, or long-term bridge to transplantation.

\section{Patient selection}

The criteria used to select patients for ECPR vary between institutions. Some centers will deploy mechanical support only in children who suffer cardiac arrest in the operating theater, cardiac catheterization laboratory, or in the ICU. Others will offer it to children who suffer out-of-hospital cardiac arrest or in the emergency department or general wards. Ideally, in intensive care units with large ECLS programs, a decision about candidacy for ECPR should be made in every patient, as well as a plan for the type of support, cannula size, and site. This is especially important in chronically ill patients whose long-term outcome might be poor and the risk of complications too high.

In an ELSO registry review of all reported cases between 1992 and 2005, $73 \%$ of children who received ECPR were cardiac patients, $8 \%$ had sepsis, and $6 \%$ had respiratory failure and $2 \%$ accidental injury [14]. The majority of cardiac patients requiring ECPR were newborns ( $83 \%$ ). Prematurity and weight $<3 \mathrm{~kg}$ were associated with worse outcomes after ECPR, presumably because of the high risk of neurological sequelae related to brain immaturity [57•]. In one case series, survival after ECPR in cardiac patients $<3 \mathrm{~kg}$ was only $25 \%$ compared to $35 \%$ in the group who was semielectively cannulated [58]. In a recent, single-center review of cardiac patients receiving ECPR [59], survival rates varied based on the underlying disease, with patients supported for cardiomyopathy/myocarditis having $89 \%$ survival rate compared to $47 \%$ of the postcardiotomy group. Patients with myocarditis may develop cardiac arrest because of worsening disease or sudden arrhythmias, and this is commonly precipitated by medical intervention. For example, in a case series of 20 fulminant myocarditis patients, 7 children required $\mathrm{CPR}, 5$ of whom were cannulated onto ECMO; in 6 out of 7 cases, cardiac arrest followed medical intervention ( 2 during intubation, 2 during amiodarone administration, and 2 during cardioversion) [60]. Having an ECMO team on stand-by during high risk procedures is important in this patient group. 
ECPR in children with single ventricle physiology has been associated with higher mortality when compared to biventricular physiology ( 69 vs. $39 \%$ ) [59]. In one series, postcardiotomy ECPR in single ventricle physiology reported 1-year survival rates of $50 \%$ [61]. In another series, children with single ventricle physiology who were cannulated for shunt obstruction had much better outcomes compared to those cannulated for poor ventricular function ( 81 vs. $29 \%$ survival) [62]. There are fewer data on survival rates for ECPR in Glenn and Fontan circulations. In an early review, only two out of five patients with Fontan circulation requiring ECPR survived and one out of three for Glenn physiology [55]. In an ELSO database review, 68 patients with Fontan circulation who received mechanical support had a cardiac arrest, and only 17 survived (25\%) [63]. In a recent retrospective study, survival rates were significant higher after correction of residual lesions (35 patients out of 119); therefore, mechanical support may improve outcomes by providing cardiovascular support as a bridge to further surgery [64•]. In an ELSO database review of all patients with Glenn anastomosis supported with ECMO, survival rate to hospital discharge $(41 \%)$ was not dissimilar than overall ECMO survival for cardiac patients, but with a higher incidence of neurological complications (23\%) [56•]. This is not surprising considering the physiological implications of a cavopulmonary anastomosis in a patient undergoing conventional CPR: High cerebral venous pressures, hypoxia, and inadequate forward flow because of high intrathoracic pressures during active compressions combine to increase the risk of venous stroke, hypoxic-ischemic events, and cerebral hemorrhage. Interestingly, despite the absence of neurological injury in children with Glenn circulation receiving ECPR (9/103), only four survived to hospital discharge. This might suggest that survival is correlated to the natural history of the disease and that even in the setting of adequate support and absence of complications, ultimately the key point for good outcome remains the reversibility of the underlying trigger for cardiorespiratory failure. In this series of children with Glenn anastomosis, factors associated with mortality were need for inotropes before ECMO, longer ECMO runs, combined cardiopulmonary indications, and renal failure while on ECMO [56•].

Considerable debate still occurs on the role of ECPR in the setting of nonprimary cardiac diagnoses-in septic shock, for example. Data from our center report an overall survival rate of $55 \%$ in patients receiving CPR during cannulation [53]. Improved outcomes may be

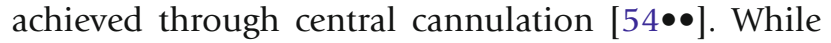

good survival after ECPR for sepsis can be achieved, much worse outcomes have been described for medical patients on mechanical support. In a case series by Morris et al. [65], survival after ECPR differed between patients with a cardiac diagnosis (44\%) and those with a noncardiac diagnosis $(9.5 \%)$. It can be difficult to decide emergently whether the patient is a good ECPR candidate in the setting of an arrest. Cardiac arrest is usually sudden and unanticipated, in which case ECPR can be used as a bridge to buy time to investigate and correct the etiology and allow the parents to try and come to terms with their child's critical illness.

Interestingly, while many ECPR studies focus on populations with cardiac disease, primary respiratory disease remains the most common cause for cardiac arrest in the pediatric population. The early use of ECMO in severe respiratory failure improves patient outcome by limiting lung injury and also allowing less fluid administration to patients as a result of the negative circulatory effects of mechanical ventilation.

Additional indications for ECPR other than cardiac disease include poisoning [66, 67], blunt chest trauma [68, 69], electrolyte disturbances [70], hypothermia $[26 \bullet, 27,71]$, and anaphylaxis [72]. Extrapolating clear guidelines on the basis of these brief reports is difficult, and candidacy for ECPR must be established on a caseby-case basis.

\section{Hypothermia and ECPR}

The use of therapeutic hypothermia (TH) for neuroprotection following ECPR is controversial. Some centers have used it routinely since the 1990s $[11,41]$ while others have not $[10,59]$. A recent review concluded that TH may be of benefit in adolescents who remain comatose after resuscitation from witnessed, out-of-hospital, ventricular fibrillation cardiac arrest, and could be considered for children who remain comatose after resuscitation from cardiac arrest [73]. The outcome data for $\mathrm{TH}$ $\left(32-34{ }^{\circ} \mathrm{C}\right)$ following cardiac arrest are difficult to interpret because of the large number of underlying conditions which precipitate cardiac arrest, variably slow times to achieve the target temperature, and variations in the speed and timing of rewarming. ECPR can bypass some of these obstacles by facilitating rapid cooling and maintenance of hypothermia, as well as permitting a more controlled rate of rewarming, when compared to standard measures. In one small retrospective series, survival rates at discharge for children with asphyxial IHCA and OHCA were higher in the hypothermia group [74]. Nonetheless, the evidence for TH in children 
remains limited [75]. In 2013, one study randomised newborns needing ECMO to normothermia or $\mathrm{TH}$; the results of the study showed no difference in outcome at 2 years, with slightly higher risk for adverse events in the

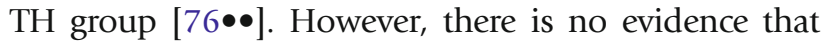
these results are applicable to older children. Although there are some encouraging data from animal studies showing improved survival and outcome with $\mathrm{TH}[77$, 78], a recent large randomised trial in adults showed no difference in outcome for OHCA patients [79]. In another randomised trial, there was no difference in survival or neurological outcome after rapid cooling prehospital admission [80]. While in the first trial cooling was delayed and rewarming was very rapid, in the second, there was no comment regarding rewarming practices. While TH may be controversial, avoiding hyperthermia with controlled temperature management is generally regarded as a standard of care [81]. The major problem with most studies is the lack of ability and understanding on the correct use of hypothermia; patient temperature needs to be $<33{ }^{\circ} \mathrm{C}$ within $4 \mathrm{~h}$, TH should continue for $48-72 \mathrm{~h}$, and rewarming should be very gradual and physiologically based [82].

\section{Rapid deployment versus early deployment}

There is no doubt that the training for and an introduction of a rapid deployment system for ECMO will decrease times to institute ECPR and potentially improve survival [45]. Hence, if rapid deployment ECMO can reduce the period of minimal or no cardiac output and end-organ damage, then early mechanical support might improve outcomes. As ECMO becomes safer, more familiar, and easier to manage, it may be considered earlier in the management strategy of critically ill children. In a review of all complications in the neonatal group supported with $\mathrm{ECMO}$, the presence of a cardiac arrest pre-ECMO was associated with worse outcome $[83 \bullet, 84,85]$. In the presence of progressive respiratory failure and hypoxia leading to cardiac arrest (e.g., ARDS, asthma, meconium aspiration, congenital diaphragmatic hernia), early venovenous support could improve outcomes, with low risk of complications and good overall survival. Moreover, there is some evidence that venovenous ECMO might also be useful in selected cardiac patients. Further analysis is needed to better define the correct indications for this group of patients [86]. In postcardiotomy patients, progressive cardiac dysfunction with raising lactate, falling mixed venous saturation, and escalating inotropes should trigger early discussion and consideration of mechanical support. Exact figures that should trigger the initiation of ECMO (e.g., oxygenation index, $\mathrm{PaO}_{2} / \mathrm{FiO}_{2}$ ratio, $\mathrm{SvO}_{2}$, lactate, inotrope scores) have not been established, and the decision to initiate ECMO preemptively relies on clinical experience and the quality of the local ECLS program. For patients with borderline cardiac output and high inotropic scores, cardiac surgeons and intensivists should always discuss initiating mechanical support in the operating theatre. In fact, improved outcomes have been described for children cannulated onto ECMO in the operating room compared to support later in the postoperative period $[84,85,87]$.

\section{Outcome}

The ELSO registry is a very useful source of information, but the standard outcome measure is survival to hospital discharge. This has several limitations, including that late deaths and long-term neurological morbidity are not captured, and it is hard to control for the enormous global variability in technology, clinical management strategies, anticoagulation, patient selection, cannulation strategies, and follow-up. A very comprehensive review of the literature examining ECMO-related outcomes in cardiac patients and children requiring ECPR was published in 2012 [88••]. Data from the ELSO registry from 1989 until January 2014 show survival after ECPR in $40 \%$ (388/980) of neonates and $41 \%$ in the pediatric group (840/2071) [89]. Noncardiac indications, kidney injury on ECMO and need for RRT, lower pH on ECMO, and development of neurologic complications negatively impact survival. The duration of CPR relates to mortality in only two studies $[41,90]$. In a recent study, neurologic complications occurred in $20 \%$ and were associated with higher mortality, especially in the group receiving ECPR (OR, 1.7) [83•]. In another study, both short- and longterm survivals after ECPR in cardiac patients were very high (75 and $70 \%$, respectively), possibly related to a reduction in complications (less bleeding, acidosis, and renal failure), higher blood flows, and less delay in decannulation. Encouragingly, $89 \%$ were normal or had mild disability at follow-up (1.9 years) [91].

Fewer data are available on functional outcomes and late mortality. In a review of the ECPR literature from 2000 to 2011, overall functional outcomes were good, with normal or mild dysfunction in $79 \%$ of children

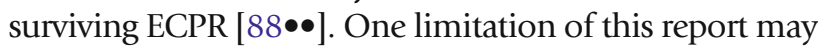
be publication bias, where only high volume centers with excellent outcomes publish their results. Another report, in which $49 \%$ of the survival group received ECPR, 
assessed the quality of life after ECMO. Physical summary scores were lower than the general American population and similar to those of children with congenital heart disease, while the psychosocial ones were similar to the general population [92]. It remains debatable if the lower physical scores reflect underlying cardiac disease history or are related to mechanical support. Regardless, good quality of life can be achieved following ECPR. However, late mortality does occur, even in patients who have seemingly recovered from their initial illness. A large series from the UK found that late mortality (beyond 90 days following ECMO) was $6.2 \%$, with a survival rate at 5 years varying between $32 \%$ for the cardiac population and $88 \%$ for meconium aspiration [93•]. Long-term follow-up and more widespread research are needed to better understand what factors contribute to the future of patients we currently support with ECMO, or if there is a group of patients where very close follow-up and prevention of deterioration might reduce late mortality, especially in the cardiac population.

\section{Conclusions}

The availability of ECMO for children in cardiac arrest is becoming a standard of care for tertiary paediatric intensive care units in high-income countries. Reduction of end-organ failure and its impact on outcome can be achieved by good CPR and prompt consideration of mechanical support. Patient selection, cannulation strategy, mode of support, circuit management, and avoidance of complications are key factors for successful outcomes. Overall survival and long-term neurological outcomes are influenced by the natural history of the primary disease, the timeliness of ECMO initiation, and the risks of iatrogenic complications. Prompt identification of the deteriorating patient, early reversal of shock and organ failure, and rapid deployment of ECMO without ECPR should be the focus to improve survival and quality of life.

\section{Compliance with Ethics Guidelines}

\section{Conflict of Interest}

Roberto Chiletti, Warwick Butt, and Graeme MacLaren declare that they have no conflicts of interest.

Human and Animal Rights and Informed Consent

This article does not contain any studies with human or animal subjects performed by any of the authors.

\section{References and Recommended Reading}

Papers of particular interest, published recently have been highlighted as:

- Of importance

$\bullet$ Of major importance

1. Ramirez M. Multiple organ dysfunction syndrome. Curr Probl Pediatr Adolesc Health Care. 2013;43(10):273-7.

2. Proulx F et al. The pediatric multiple organ dysfunction syndrome. Pediatr Crit Care Med. 2009;10(1):12-22.

3. ELSO. ECMO: extracorporeal cardiopulmonary support in critical care 4th Edition.
4. Butt W, Maclaren G. Extracorporeal membrane oxygenation. F1000Prime Rep. 2013; 5: 55.

5. Lopez-Herce J et al. Factors associated with mortality in pediatric in-hospital cardiac arrest: a prospective multicenter multinational observational study. Intensive Care Med. 2013;39(2):309-18. 
6. $\quad$ Girotra S et al. Survival trends in pediatric in-hospital cardiac arrests: an analysis from Get with the Guidelines-resuscitation. Circ Cardiovasc Qual Outcomes. 2013;6(1):42-9.

This retrospective review reports improved survival to hospital discharge in the last decade for in-hospital cardiac arrest in children and no difference in neurological disability.

7. Gillis J et al. Results of inpatient pediatric resuscitation. Crit Care Med. 1986;14(5):469-71.

8. Michiels EA et al. Long-term outcomes following pediatric out-of-hospital cardiac arrest* ${ }^{*}$ Pediatr Crit Care Med. 2013;14(8):755-60.

9. $\quad$ Sivarajan VB et al. Improved outcomes of paediatric extracorporeal support associated with technology change. Interact Cardiovasc Thorac Surg. 2010;11(4):400-5.

This study demontrates improved outcome secondary to technological changes in ECMO circuit from 1988 until 2006.

10. Huang SC et al. Eleven years of experience with extracorporeal cardiopulmonary resuscitation for paediatric patients with in-hospital cardiac arrest. Resuscitation. 2012;83(6):710-4.

11. Kane DA et al. Rapid-response extracorporeal membrane oxygenation to support cardiopulmonary resuscitation in children with cardiac disease. Circulation. 2010;122 Suppl 11:S241-8.

12. Matos MR et al. Response to letters regarding article, "Duration of cardiopulmonary resuscitation and illness category impact survival and neurologic outcomes for in-hospital pediatric cardiac arrests". Circulation. 2013;128(7):e102-3.

13. Chan T et al. Survival after extracorporeal cardiopulmonary resuscitation in infants and children with heart disease. J Thorac Cardiovasc Surg. 2008;136(4):98492.

14. Thiagarajan RR et al. Extracorporeal membrane oxygenation to aid cardiopulmonary resuscitation in infants and children. Circulation. 2007;116(15):1693700 .

15. Huang SC et al. Extracorporeal membrane oxygenation rescue for cardiopulmonary resuscitation in pediatric patients. Crit Care Med. 2008;36(5):1607-13.

16. Tajik M, Cardarelli MG. Extracorporeal membrane oxygenation after cardiac arrest in children: what do we know? Eur J Cardiothorac Surg. 2008;33(3):409-17.

17. Raymond TT et al. Outcomes among neonates, infants, and children after extracorporeal cardiopulmonary resuscitation for refractory inhospital pediatric cardiac arrest: a report from the National Registry of Cardiopulmonary Resuscitation. Pediatr Crit Care Med. 2010;11(3):362-71.

18. Zwiers AJ et al. Acute kidney injury is a frequent complication in critically ill neonates receiving extracorporeal membrane oxygenation: a 14-year cohort study. Crit Care. 2013;17(4):R151.

19. Young KD et al. A prospective, population-based study of the epidemiology and outcome of out-of-hospital pediatric cardiopulmonary arrest. Pediatrics. 2004;114(1):157-64.
20. Avalli L et al. Favourable survival of in-hospital compared to out-of-hospital refractory cardiac arrest patients treated with extracorporeal membrane oxygenation: an Italian tertiary care centre experience. Resuscitation. 2012;83(5):579-83.

This study shows how survival after ECPR for out-of-hospital cardiac arrest in adults rema

ins poor when compared to in-hospital cardiac arrest.

21. Kagawa E et al. Should we emergently revascularize occluded coronaries for cardiac arrest?: rapid-response extracorporeal membrane oxygenation and intra-arrest percutaneous coronary intervention. Circulation. 2012;126(13):1605-13.

22. Le Guen $M$ et al. Extracorporeal life support following out-of-hospital refractory cardiac arrest. Crit Care. 2011;15(1):R29.

23. Ferrari $\mathrm{M}$ et al. Better outcome after cardiopulmonary resuscitation using percutaneous emergency circulatory support in non-coronary patients compared to those with myocardial infarction. Acute Card Care. 2011;13(1):30-4.

24.• Morimura N et al. Extracorporeal cardiopulmonary resuscitation for out-of-hospital cardiac arrest: a review of the Japanese literature. Resuscitation. 2011;82(1):10-4.

In this review of the Japanese experience, improved outcomes after ECPR for out-of-hospital cardiac arrest are described.

25. Sakamoto T et al. Extracorporeal cardiopulmonary resuscitation versus conventional cardiopulmonary resuscitation in adults with out-of-hospital cardiac arrest: a prospective observational study. Resuscitation. 2014;85(6):762-8.

26. Sawamoto K et al. Outcome from severe accidental hypothermia with cardiac arrest resuscitated with extracorporeal cardiopulmonary resuscitation. Am J Emerg Med. 2014;32(4):320-4.

This study shows how hypothermic cardiac arrest treated with ECPR has better outcomes when non-asphyxial.

27. Morita $S$ et al. Efficacy of portable and percutaneous cardiopulmonary bypass rewarming versus that of conventional internal rewarming for patients with accidental deep hypothermia. Crit Care Med. 2011;39(5):1064-8.

28. Silfvast T, Pettila V. Outcome from severe accidental hypothermia in Southern Finland-a 10-year review. Resuscitation. 2003;59(3):285-90.

29. Farstad $\mathrm{M}$ et al. Rewarming from accidental hypothermia by extracorporeal circulation. A retrospective study. Eur J Cardiothorac Surg. 2001;20(1):58-64.

30. Peek GJ, Firmin RK. The inflammatory and coagulative response to prolonged extracorporeal membrane oxygenation. ASAIO J. 1999;45(4):250-63.

31. Lequier L et al. Extracorporeal membrane oxygenation circuitry. Pediatr Crit Care Med. 2013;14(5 Suppl 1):S7-S12.

32. Megarbane B et al. Usefulness of routine laboratory parameters in the decision to treat refractory cardiac arrest with extracorporeal life support. Resuscitation. 2011;82(9):1154-61. 
33. Lawson $\mathrm{S}$ et al. Neonatal extracorporeal membrane oxygenation devices, techniques and team roles: 2011 survey results of the United States' Extracorporeal Life Support Organization Centers. J Extra Corp Technol. 2011;43(4):236-44.

34. Reed-Thurston D et al. Pediatric and neonatal extracorporeal life support technology component utilization: are US clinicians implementing new technology? Artif Organs. 2012;36(7):607-15.

35. Barrett CS et al. Pediatric ECMO outcomes: comparison of centrifugal versus roller blood pumps using propensity score matching. ASAIO J. 2013;59(2):14551.

Propensity score-matching of patients supported with centrifulgal vs. roller pump. This study reports higher risk of complications with centrifugal pumps but without difference in survival.

36. Lou $\mathrm{S}$ et al. Hemolysis in pediatric patients receiving centrifugal-pump extracorporeal membrane oxygenation: prevalence, risk factors, and outcomes. Crit Care Med. 2014;42(5):1213-20.

37. Bottrell S et al. A comparison study of haemolysis production in three contemporary centrifugal pumps. Perfusion, 2014.

38. Karimova A et al. A wet-primed extracorporeal membrane oxygenation circuit with hollow-fiber membrane oxygenator maintains adequate function for use during cardiopulmonary resuscitation after 2 weeks on standby. Crit Care Med. 2005;33(7):1572-6.

39. Naso F et al. Wet-priming extracorporeal membrane oxygenation device maintains sterility for up to 35 days of follow-up. Perfusion. 2013;28(3):208-13.

This study proves that sterility is mantained in wet pre-primed ECMO circuits.

40. Duncan BW et al. Use of rapid-deployment extracorporeal membrane oxygenation for the resuscitation of pediatric patients with heart disease after cardiac arrest. J Thorac Cardiovasc Surg. 1998;116(2):305-11.

41. Sivarajan VB et al. Duration of resuscitation prior to rescue extracorporeal membrane oxygenation impacts outcome in children with heart disease. Intensive Care Med. 2011;37(5):853-60.

42. Turek JW et al. Outcomes before and after implementation of a pediatric rapid-response extracorporeal membrane oxygenation program. Ann Thorac Surg. 2013;95(6):2140-6.

In this study neurological outcomes but not survival are improved after implementation of a rapid response ECMO program.

43.• Chai PJ et al. Extracorporeal cardiopulmonary resuscitation for post-operative cardiac arrest: indications, techniques, controversies, and early results-what is known (and unknown). Cardiol Young. 2011;21 Suppl 2:109-17.

This review analyzes all the previous published literature regarding ECPR in the post-cardiotomy population.

44. Alsoufi B et al. Results of rapid-response extracorporeal cardiopulmonary resuscitation in children with refractory cardiac arrest following cardiac surgery. Eur J Cardiothorac Surg. 2014;45(2):268-75.

In this study of children requiring ECPR post-cardiotomy, survival is correlated to early mechanical support and reversal of organ falure, and correction of residual lesions.

45. Su L et al. Implementation of an extracorporeal cardiopulmonary resuscitation simulation program reduces extracorporeal cardiopulmonary resuscitation times in real patients*. Pediatr Crit Care Med. 2014;15(9):856-60.

46. Ghez $\mathrm{O}$ et al. Absence of rapid deployment extracorporeal membrane oxygenation (ECMO) team does not preclude resuscitation ecmo in pediatric cardiac patients with good results. ASAIO J. 2007;53(6):692-5.

47.• Guerguerian AM et al. Setup and maintenance of extracorporeal life support programs. Pediatr Crit Care Med. 2013;14(5 Suppl 1):S84-93.

Detailed analysis on how to set up and maintain an ECLS program.

48. Karamlou $\mathrm{T}$ et al. Increased extracorporeal membrane oxygenation center case volume is associated with improved extracorporeal membrane oxygenation survival among pediatric patients. J Thorac Cardiovasc Surg. 2013;145(2):470-5.

49. $\quad$ Freeman CL et al. Pediatric and neonatal extracorporeal membrane oxygenation: does center volume impact mortality?*. Crit Care Med. 2014;42(3):512-9.

This study demontrates that higher volume centers ECMO probably have improved survival compared to low volume centers.

50. Dalton HJ, Butt WW. Extracorporeal life support: an update of Rogers' textbook of pediatric intensive care. Pediatr Crit Care Med. 2012;13(4):461-71.

51. Sivarajan VB et al. Pediatric extracorporeal life support in specialized situations. Pediatr Crit Care Med. 2013;14(5 Suppl 1):S51-61.

$52 . \bullet$ Teele SA et al. The association of carotid artery cannulation and neurologic injury in pediatric patients supported with venoarterial extracorporeal membrane oxygenation*. Pediatr Crit Care Med. 2014;15(4):355-61. In this retrospective analysis of the ELSO database, carotid cannulation is associated with higher risk of neurological injury when compared with central or femoral cannulation.

53. Maclaren G, Butt W. Extracorporeal membrane oxygenation and sepsis. Crit Care Resusc. 2007;9(1):76-80.

54.• MacLaren G et al. Central extracorporeal membrane oxygenation for refractory pediatric septic shock. Pediatr Crit Care Med. 2011;12(2):133-6.

This study demonstrates that ECMO in sepsis improves survival when associated with central cannulation.

55. Booth KL et al. Extracorporeal membrane oxygenation support of the Fontan and bidirectional Glenn circulations. Ann Thorac Surg. 2004;77(4):1341-8.

56. Jolley $\mathrm{M}$ et al. Extracorporeal membrane oxygenation in patients undergoing superior cavopulmonary anastomosis. J Thorac Cardiovasc Surg, 2014.

In this ELSO database review, survival after ECMO in cavopulmonary anastomosis has improved over time but neurological complications remain high. 
57. McMullan DM et al. Extracorporeal cardiopulmonary resuscitation outcomes in term and premature neonates*. Pediatr Crit Care Med. 2014;15(1):e9-e16.

In this study of neonates supported wth ECMO, lower gestational age, need for ECPR and development of neurological complications are associated with higher mortality.

58. Bhat $\mathrm{P}$ et al. Outcomes of infants weighing three kilograms or less requiring extracorporeal membrane oxygenation after cardiac surgery. Ann Thorac Surg. 2013;95(2):656-61.

59. Wolf MJ et al. Extracorporeal cardiopulmonary resuscitation for pediatric cardiac patients. Ann Thorac Surg 2012;94(3):874-9.

60. Teele SA et al. Management and outcomes in pediatric patients presenting with acute fulminant myocarditis. J Pediatr. 2011;158(4):638-643.e1.

61. Polimenakos AC et al. Post-cardiotomy extracorporeal cardiopulmonary resuscitation in neonates with complex single ventricle: analysis of outcomes. Eur J Cardiothorac Surg. 2011;40(6):1396-405. discussion 1405.

62. Allan CK et al. Indication for initiation of mechanical circulatory support impacts survival of infants with shunted single-ventricle circulation supported with extracorporeal membrane oxygenation. J Thorac Cardiovasc Surg. 2007;133(3):660-7.

63. Rood KL et al. Extracorporeal membrane oxygenation support after the Fontan operation. J Thorac Cardiovasc Surg. 2011;142(3):504-10.

64. Agarwal HS et al. Residual lesions in postoperative pediatric cardiac surgery patients receiving extracorporeal membrane oxygenation support. J Thorac Cardiovasc Surg. 2014;147(1):434-41.

This review demonstrates that residual lesions are present in one quarter of children supported with ECMO; improved outcomes can be achieved through early detection and correction of these lesions.

65. Morris MC, Wernovsky G, Nadkarni VM. Survival outcomes after extracorporeal cardiopulmonary resuscitation instituted during active chest compressions following refractory in-hospital pediatric cardiac arrest. Pediatr Crit Care Med. 2004;5(5):440-6.

66. Felton TW et al. Life-threatening pulmonary hypertension and right ventricular failure complicating calcium and phosphate replacement in the intensive care unit. Anaesthesia. 2006;61(1):49-53.

67. Haas NA et al. ECMO for cardiac rescue in a neonate with accidental amiodarone overdose. Clin Res Cardiol. 2008;97(12):878-81.

68. Incagnoli $\mathrm{P}$ et al. Pulmonary resection and ECMO: a salvage therapy for penetrating lung trauma. Ann Fr Anesth Reanim. 2012;31(7-8):641-3.

69. Ballouhey Q et al. Benefits of extracorporeal membrane oxygenation for major blunt tracheobronchial trauma in the paediatric age group. Eur J Cardiothorac Surg. 2013;43(4):864-5.

70. Vivekananthan $\mathrm{R}$ et al. ECPR for prolonged paediatric cardiac arrest. Anaesth Intensive Care. 2014;42(1):147-8.
71. Boue Y et al. Neurologic recovery from profound accidental hypothermia after 5 hours of cardiopulmonary resuscitation. Crit Care Med. 2014;42(2):e167-70.

72. Chan-Dominy A. et al. Extracorporeal membrane modality conversions. Perfusion, 2014.

73. Scholefield BR et al. Should children who have a cardiac arrest be treated with therapeutic hypothermia? BMJ. 2014;348:f7672.

74. Lin JJ et al. Therapeutic hypothermia associated with increased survival after resuscitation in children. Pediatr Neurol. 2013;48(4):285-90.

75. Scholefield B et al. Hypothermia for neuroprotection in children after cardiopulmonary arrest. Cochrane Database Syst Rev. 2013;2:Cd009442.

76.• Field D et al. Neonatal ECMO study of temperature (NEST): a randomized controlled trial. Pediatrics. 2013;132(5):e1247-56.

In this randomized controlled trial, there was no improved neurological outcome at 2 years follow-up for neonates who received therapeutic hypothermia while on ECMO.

77. Zhao H, Chen Y, Jin Y. The effect of therapeutic hypothermia after cardiopulmonary resuscitation on ICAM1 and NSE levels in sudden cardiac arrest rabbits. Int J Neurosci, 2014: 1-16.

78. Sterz F et al. Mild hypothermic cardiopulmonary resuscitation improves outcome after prolonged cardiac arrest in dogs. Crit Care Med. 1991;19(3):379-89.

79. Nielsen $\mathrm{N}$ et al. Targeted temperature management at 33 degrees $\mathrm{C}$ versus 36 degrees $\mathrm{C}$ after cardiac arrest. $\mathrm{N}$ Engl J Med. 2013;369(23):2197-206.

80. Kim F et al. Effect of prehospital induction of mild hypothermia on survival and neurological status among adults with cardiac arrest: a randomized clinical trial. JAMA. 2014;311(1):45-52.

81. Moler FW et al. Rationale, timeline, study design, and protocol overview of the therapeutic hypothermia after pediatric cardiac arrest trials. Pediatr Crit Care Med. 2013;14(7):e304-15.

82. Shann F. Hypothermia for traumatic brain injury: how soon, how cold, and how long? Lancet. 2003;362(9400):1950-1.

83. Polito A et al. Neurologic complications in neonates supported with extracorporeal membrane oxygenation. An analysis of ELSO registry data. Intensive Care Med. 2013;39(9):1594-601.

In this ELSO database review, factors associated with development of neurological complications in neonates supported with ECMO were low gestational age and birth weight, need for ECPR and use of VA-ECMO.

84. Barrett CS et al. Neurological injury after extracorporeal membrane oxygenation use to aid pediatric cardiopulmonary resuscitation. Pediatr Crit Care Med. 2009;10(4):445-51.

85. Minneci PC et al. Factors associated with mortality in pediatric patients requiring extracorporeal life support for severe pneumonia. Pediatr Crit Care Med. 2013;14(1):e26-33.

86. Kim $\mathrm{K}$ et al. Use of venovenous extracorporeal life support in pediatric patients for cardiac indications: a 
review of the extracorporeal life support organization registry. Pediatr Crit Care Med. 2012;13(3):285-9.

87. Mesher AL, McMullan DM. Extracorporeal life support for the neonatal cardiac patient: outcomes and new directions. Semin Perinatol. 2014;38(2):97-103.

88.•• Joffe AR, Lequier L, Robertson CM. Pediatric outcomes after extracorporeal membrane oxygenation for cardiac disease and for cardiac arrest: a review. ASAIO J. 2012;58(4):297-310.

This review identifies non-cardiac disease, renal dysfunction, neurological complications and $\mathrm{pH}$ on ECMO as factors associated with worse outcome after ECPR.

89. ELSO. Extracorporeal membrane oxygenation registry of the extracorporeal life support organization. Ann Arbor: ELSO; 2014.

90. Kumar TK et al. Extracorporeal membrane oxygenation in postcardiotomy patients: factors influencing outcome. J Thorac Cardiovasc Surg. 2010;140(2):330336.e2.

91. Chrysostomou C et al. Short- and intermediate-term survival after extracorporeal membrane oxygenation in children with cardiac disease. J Thorac Cardiovasc Surg. 2013;146(2):317-25.

92. Costello JM et al. Quality of life of pediatric cardiac patients who previously required extracorporeal membrane oxygenation. Pediatr Crit Care Med. 2012;13(4):428-34.

93. Iguchi A et al. Long-term survival outcomes and causes of late death in neonates, infants, and children treated with extracorporeal life support. Pediatr Crit Care Med. 2013;14(6):580-6.

This single center study describes late mortality after ECMO support in patients with congenital diaphragmatic hernia and cardiac disease. 